\title{
Prevalence, risk factors and prognostic role of anxiety and depression in surgical gastric cancer patients
}

\author{
Lijuan Han \\ Department of Surgery, The 2nd Affiliated Hospital of Harbin Medical University, Harbin 150001, China \\ Correspondence to: Lijuan Han. Department of Surgery, The 2nd Affiliated Hospital of Harbin Medical University, 246 Xuefu Road, Harbin 150001, \\ China. Email: xun48382403@163.com.
}

\begin{abstract}
Background: The present study was conducted to explore the prevalence, risk factors and prognostic role of anxiety and depression in surgical gastric cancer patients.

Methods: Totally 200 gastric cancer patients who underwent surgical resection were enrolled and their Hospital Anxiety and Depression Scale (HADS) scores were assessed before surgery. Besides, 200 healthy controls were enrolled with their HADS scores assessed at enrollment.
\end{abstract}

Results: The HADS-anxiety score $(7.4 \pm 3.8$ vs. $4.7 \pm 2.8, \mathrm{P}<0.001)$, prevalence $(42.5 \%$ vs. $13.5 \%, \mathrm{P}<0.001)$ and severity $(\mathrm{P}=0.002)$ of anxiety were greatly increased in gastric cancer patients compared with healthy controls. And HADS-depression score $(6.9 \pm 3.5$ vs. $4.2 \pm 2.6, \mathrm{P}<0.001)$, prevalence $(33.5 \%$ vs. $10.0 \%, \mathrm{P}<0.001)$ and severity $(\mathrm{P}=0.001)$ of depression were also dramatically elevated in gastric cancer patients compared with healthy controls. Regarding the factors predicting anxiety/depression risks in gastric cancer patients, gender (female) $(\mathrm{P}=0.003)$, diabetes $(\mathrm{P}<0.001)$, higher pathological grade $(\mathrm{P}=0.005)$, larger tumor size $(\mathrm{P}=0.044)$ and higher $\mathrm{T}$ stage $(\mathrm{P}=0.017)$ were independent predictive factors for higher risk of anxiety, and age ( $>60$ years) $(\mathrm{P}=0.025)$, gender (female) $(\mathrm{P}=0.004)$, hyperlipidemia $(\mathrm{P}=0.039)$, diabetes $(\mathrm{P}<0.001)$ and higher TNM stage $(\mathrm{P}=0.003)$ were independent predictive factors for higher risk of depression. Most importantly, anxiety/depression and the severity of anxiety/depression were all negatively associated with disease-free survival as well as overall survival (all $\mathrm{P}<0.05)$ in surgical gastric cancer patients.

Conclusions: Anxiety/depression are common and severe, which predicts unfavorable prognosis in gastric cancer patients underwent surgery, suggesting the necessity of psychological care post-surgery.

Keywords: Anxiety; depression; gastric cancer patients; postoperative; survival

Submitted Aug 22, 2019. Accepted for publication Dec 12, 2019.

doi: $10.21037 /$ tcr.2020.01.11

View this article at: http://dx.doi.org/10.21037/tcr.2020.01.11

\section{Introduction}

Gastric cancer ranks as the fifth most frequently diagnosed cancer and the third leading cause of cancer-related mortality globally, with over 1,00,000 new cases and estimated 783,000 deaths worldwide in $2018(1,2)$. There have been considerable improvements in the surgical techniques and progression in traditional radiotherapy, chemotherapy and corresponding neoadjuvant therapy, thus the 5-year survival rate of gastric cancer patients had been prolonged $(3,4)$. However, gastric cancer patients following the gastrectomy are especially vulnerable to the physiology problems, such as pain, reflux, dyspnea, dysphagia, eating restrictions, which might increase their risk of psychological problems, such as anxiety and depression (5-7). Therefore, it is essential to investigate the predictive factors of anxiety/ depression as well as explore the influence of anxiety and depression on prognosis in surgical gastric cancer patients.

Previous studies uncover several risk factors for psychological distress in patients with digestive cancers (8-10). For example, in colon cancer patients underwent proctocolectomy, pre-operative corticosteroid use, female gender, tobacco usage and pouch inflammation are independently associated with anxiety and depression 
risk (10). And another study reveals that middle age and chemotherapy are associated with more severe anxiety, and medical treatment equipment, chemotherapy as well as long-term hospitalization are associated with more severe depression in digestive cancers patients (8). Besides, some reports illustrate that unfavorable psychological distress might have negative effect on quality of life in cancer patients, contributing to slow recovery and poor survival profiles (9). However, there is limited report of the survival influence of anxiety and depression in surgical gastric cancer patients.

Therefore, the present study was performed for the purposes as follows: (I) surgical gastric cancer patients and age/gender matched healthy controls were recruited, and the prevalence and severity of anxiety/depression between gastric cancer patients and healthy controls was compared; (II) the predictors of anxiety/depression risk was further explored in surgical gastric cancer patients; (III) the association of anxiety/depression with prognosis was detected in surgical gastric cancer patients.

\section{Methods}

\section{Participants}

This study consecutively recruited 200 gastric cancer patients who underwent surgical resection in the 2 nd Affiliated Hospital of Harbin Medical University between July 2014 and June 2017. The inclusion criteria were: (I) pathologically confirmed as primary gastric cancer; (II) clinical status is suitable for surgery; (III) scheduled for gastrectomy; (IV) age above 18 years; (V) willingness to be followed up regularly. The exclusion criteria were: (I) received anxiolytic or antidepressants treatment within 3 months before enrollment; (II) complicated with other malignancies; (III) complicated with cognitive impairments and cannot understand the questions in Hospital Anxiety and Depression Scale (HADS); (IV) complicated with uncontrolled hypertension, cardiac arrhythmia or unstable angina pectoris; (V) pregnant women or lactating women; (VI) received neo-adjuvant therapy. Besides, 200 healthy subjects who underwent physical examination in our hospital between July 2016 and June 2017 were enrolled as healthy controls. The healthy controls were defined as healthy individuals who showed no obvious abnormalities of biochemical indexes, no liver or kidney dysfunction and no history of malignancies, uncontrolled hypertension, cardiac arrhythmia or unstable angina pectoris.

\section{Ethics}

This study was approved by the Ethics Committee of the 2nd Affiliated Hospital of Harbin Medical University and conducted in accord with the Declaration of Helsinki. All participants signed the informed consents before they participated in the study.

\section{Data collection}

The clinical data of gastric cancer patients were recorded, which included of: (I) socio-demographic characteristics (age, gender, nationality, smoke, drink, marry status, employment status before surgery and level of education); (II) common complications (hypertension, hyperlipidemia and diabetes); (III) helicobacter pylori infection status; (IV) tumor features (tumor location, tumor size, pathological grade, T stage, $\mathrm{N}$ stage and TNM stage); (V) adjuvant therapy (chemotherapy and radiotherapy).

\section{Anxiety and depression assessment}

The HADS was employed to assess the anxiety and depression of the participants. For the gastric cancer patients, the HADS was assessed before surgery. And for healthy controls, the HADS was assessed at enrollment. A total of 14 items were included in HADS, seven items reflect anxiety (HADS-A) and the other 7 items indicate depression (HADS-D) (11). The total scores on each subscale ranging from 0 to 21 , and with higher scores corresponding to greater respective symptom severity $(<8$ no anxiety or depression, 8-10 mild anxiety or depression, 11-14 moderate anxiety or depression, $>14$ severe anxiety or depression) (11).

\section{Follow-up}

After surgery, intensive follow-up was carried out for all patients, and according to their clinical situation appropriate treatment regimens were given by attending physicians. The last follow-up date was 2019/06/30, and the median followup was 36.0 months (range, 3.0-59.0 months). Diseasefree survival (DFS) was defined as the duration from the date of resection to the date of relapse or death. Overall survival (OS) was defined as the duration from the date of resection to the date of death. All patients were included in the final analysis, and for the patients who lost follow-up or withdrew from the study, DFS and OS were calculated from 


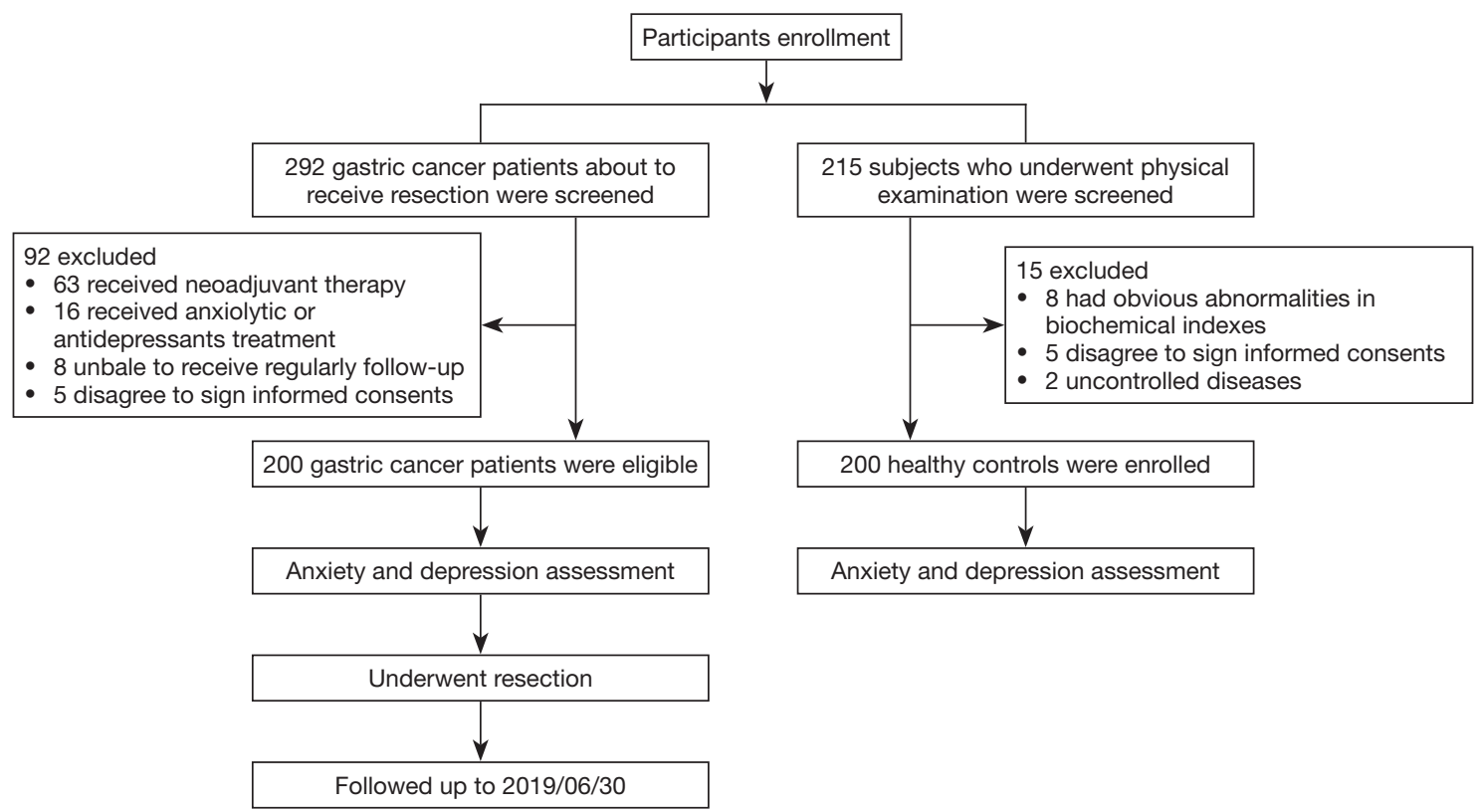

Figure 1 Study flow.

the date of resection to the date of last visit or last known to be alive.

\section{Statistical analysis}

Data was expressed as mean and standard deviation (SD) or count (percentage). Objective 1: (I) the comparison of HADS-A and HADS-D score between gastric cancer patients and healthy controls was determined by student's $t$-test; (II) the comparison of anxiety/depression rate between gastric cancer patients and healthy controls was detected by Chi-square test; (III) the comparison of anxiety/ depression severity was determined by Wilcoxon rank sum test. Objective 2: factors affecting anxiety or depression in gastric cancer patients was analyzed by univariate and forward stepwise multivariate logistic regression model. Objective 3: DFS and OS were displayed by KaplanMeier curves, and the correlation of DFS/OS with anxiety/ depression was determined by log-rank test. All analyses were performed with the use of SPSS 22.0 software (IBM, Chicago, IL, USA) and figures were made using GraphPad Prism 7.01 software (GraphPad Software, San Diego, California, USA). $\mathrm{P}$ value $<0.05$ was considered significant.

\section{Results}

\section{Study flow}

After participants enrollment, totally 292 gastric cancer patients who were about to receive resection were screened, and 92 of them were excluded (including 63 receiving neoadjuvant therapy, 16 receiving anxiolytic or antidepressants treatment, 8 unable to receive regularly follow-up, and 5 who disagreed to sign informed consents) (Figure 1). The remaining 200 gastric cancer patients were eligible for anxiety and depression assessment. After resection surgery, follow-up was continued for all gastric cancer patients until 2019/06/30. As for control group, 215 subjects who underwent physical examination were screened, 15 of them were excluded (including 8 who had obvious abnormalities in biochemical indexes, 5 who disagreed to sign informed consents, and 2 with uncontrolled diseases). The remaining 200 healthy controls were enrolled for anxiety and depression assessment.

\section{Clinical characteristics}

The mean age of gastric cancer patients $(\mathrm{N}=200)$ was 
$59.9 \pm 10.7$ years (Table 1 ). The majority of patients were males $(58.0 \%$ ), and $70.0 \%$ patients were married. Based on the pathological grade, the numbers of patients with G1, G2 and G3 pathological grade were 30 (15.0\%), 144 $(72.0 \%)$ and $26(13.0 \%)$ respectively. And the numbers of patients with TNM stage I, II, III were 22 (11.0\%), 84 $(42.0 \%)$ and $94(47.0 \%)$ respectively. And there were 133 (66.5\%) patients receiving adjuvant chemotherapy and 26 $(13.0 \%)$ patients receiving adjuvant radiotherapy. As for the clinical characteristics of healthy controls $(\mathrm{N}=200)$, the mean age was $59.1 \pm 11.9$ years, and the number of females and males were $91(45.5 \%)$ and $109(54.5 \%)$ respectively. There was no difference of age $(\mathrm{P}=0.495)$, gender $(\mathrm{P}=0.480)$, smoke $(\mathrm{P}=0.661)$, drink $(\mathrm{P}=0.127)$, marry status $(\mathrm{P}=0.553)$, employment status before surgery $(\mathrm{P}=0.104)$, level of education $(\mathrm{P}=0.602)$, hypertension $(\mathrm{P}=0.376)$, hyperlipidemia $(\mathrm{P}=0.485)$, diabetes $(\mathrm{P}=0.672)$ between gastric cancer patients and healthy controls. The detailed information of clinical characteristic was listed in Table 1.

\section{Comparison of anxiety and depression between gastric cancer patients and healthy controls with objective 1}

The HADS-A score was increased in gastric cancer patients $(7.4 \pm 3.8)$ compared with healthy controls $(4.7 \pm 2.8)$ $(\mathrm{P}<0.001)$ (Figure $2 A)$, and the prevalence of anxiety was greatly elevated in gastric cancer patients $(42.5 \%)$ compared with healthy controls $(13.5 \%)(\mathrm{P}<0.001)$ (Figure $2 B)$. Meanwhile, there were $9(10.6 \%), 35(41.2 \%)$ and 41 $(48.2 \%)$ gastric cancer patients with severe, moderate and mild anxiety, while $0(0.0 \%), 5(18.5 \%)$ and $22(81.5 \%)$ health controls with severe, moderate and mild anxiety, and further comparison indicated that anxiety severity in gastric cancer patients was greatly higher compared with healthy controls $(\mathrm{P}=0.002)$ (Figure $2 C)$. As for depression, the HADS-D score was $6.9 \pm 3.5$ in gastric cancer patients, which was higher compared with healthy controls $(4.2 \pm 2.6)$ $(\mathrm{P}<0.001)$ (Figure 2D), and the prevalence of depression was $33.5 \%$ in gastric cancer patients, which was increased compared with healthy controls $(10.0 \%)(\mathrm{P}<0.001)$ (Figure 2E). There were $6(9.0 \%), 28(41.8 \%)$ and 33 $(49.3 \%)$ gastric cancer patients with severe, moderate and mild depression, while there were $0(0.0 \%), 2(10.0 \%)$ and $18(90.0 \%)$ healthy controls with severe, moderate and mild depression, and further comparison revealed that depression severity was increased in gastric cancer patients compared with healthy controls $(\mathrm{P}=0.001)$ (Figure $2 F)$.

\section{Factors affecting anxiety in gastric cancer patients with objective 2}

Univariate logistic regression presented that gender (female) [odds ratio $(\mathrm{OR})=1.848, \mathrm{P}=0.035$ ], hypertension $(\mathrm{OR}$ $=1.972, \mathrm{P}=0.029)$, diabetes $(\mathrm{OR}=6.070, \mathrm{P}<0.001)$, single status $(\mathrm{OR}=2.632, \mathrm{P}=0.036)$, higher pathological grade $(\mathrm{OR}$ $=2.285, \mathrm{P}=0.005)$, larger tumor size $(>3 \mathrm{~cm})(\mathrm{OR}=2.075$, $\mathrm{P}=0.013)$, higher $\mathrm{T}$ stage $(\mathrm{OR}=2.491, \mathrm{P}=0.035)$, higher $\mathrm{N}$ stage $(\mathrm{OR}=1.376, \mathrm{P}=0.040)$ and higher $\mathrm{TNM}$ stage $(\mathrm{OR}=1.802, \mathrm{P}=0.009)$ were correlated with increased risk of anxiety in gastric cancer patients (Table 2). And forward stepwise multivariate logistic regression suggested gender (female) $(\mathrm{OR}=2.835, \mathrm{P}=0.003)$, diabetes $(\mathrm{OR}=6.977, \mathrm{P}<0.001)$, higher pathological grade $(\mathrm{OR}=2.540, \mathrm{P}=0.005)$, larger tumor size $(>3 \mathrm{~cm})(\mathrm{OR}=1.992, \mathrm{P}=0.044)$ and higher $\mathrm{T}$ stage $(\mathrm{OR}$ $=3.090, \mathrm{P}=0.017)$ were independent predictive factors for higher risk of anxiety in gastric cancer patients (Table 2).

\section{Factors affecting depression in gastric cancer patients with objective 2}

Univariate logistic regression revealed that gender (female) $(\mathrm{OR}=1.875, \mathrm{P}=0.038)$, hypertension $(\mathrm{OR}=1.965, \mathrm{P}=0.034)$, diabetes $(\mathrm{OR}=6.736, \mathrm{P}<0.001)$, divorced/widowed $(\mathrm{OR}$ $=2.368, \mathrm{P}=0.023)$, higher pathological grade $(\mathrm{OR}=2.000$, $\mathrm{P}=0.020)$, higher $\mathrm{T}$ stage $(\mathrm{OR}=2.988, \mathrm{P}=0.035)$, higher $\mathrm{N}$ stage $(\mathrm{OR}=1.487, \mathrm{P}=0.016)$, higher $\mathrm{TNM}$ stage (OR $=2.004, \mathrm{P}=0.005)$ were correlated with higher risk of depression in gastric cancer patients (Table 3). Followed forward stepwise multivariate logistic regression indicated that age ( $>60$ years) $(\mathrm{OR}=0.452, \mathrm{P}=0.025)$, gender (female) $(\mathrm{OR}=2.746, \mathrm{P}=0.004)$, hyperlipidemia $(\mathrm{OR}=2.218$, $\mathrm{P}=0.039)$, diabetes $(\mathrm{OR}=7.112, \mathrm{P}<0.001)$ and higher $\mathrm{TNM}$ stage $(\mathrm{OR}=2.241, \mathrm{P}=0.003)$ were independent predictive factors for higher risk of depression in gastric cancer patients (Table 2).

\section{Correlation of anxiety/depression with DFS in gastric cancer patients with objective 3}

According to the cut-off of 8 assessed by HADS-A, all patients were divided into patients with anxiety and those without anxiety (Figure $3 A$ ). DFS was decreased in patients with anxiety compared with patients without anxiety $(\mathrm{P}<0.001)$. In addition, patients with severe anxiety had the shortest DFS, followed by patients with moderate anxiety, 
Table 1 Clinical characteristics

\begin{tabular}{|c|c|c|c|}
\hline Items & Gastric cancer patients $(\mathrm{N}=200)$ & Healthy controls $(\mathrm{N}=200)$ & $P$ value \\
\hline Gender, No. (\%) & & & 0.480 \\
\hline Female & $84(42.0)$ & $91(45.5)$ & \\
\hline Male & $116(58.0)$ & $109(54.5)$ & \\
\hline Drink, No. (\%) & $67(33.5)$ & $53(26.5)$ & 0.127 \\
\hline Marry status, No. (\%) & & & 0.553 \\
\hline Married & $140(70.0)$ & $137(68.5)$ & \\
\hline Divorced/widowed & $37(18.5)$ & $33(16.5)$ & \\
\hline Unemployed & $126(63.0)$ & $110(55.0)$ & \\
\hline Employed & $74(37.0)$ & $90(45.0)$ & \\
\hline Level of education, No. (\%) & & & 0.602 \\
\hline Primary school or less & $18(9.0)$ & $13(6.5)$ & \\
\hline High school & $92(46.0)$ & $100(50.0)$ & \\
\hline Undergraduate & $69(34.5)$ & $62(31.0)$ & \\
\hline Graduate or above & $21(10.5)$ & $25(12.5)$ & \\
\hline Hypertension, No. (\%) & $61(30.5)$ & $53(26.5)$ & 0.376 \\
\hline Tumor location, No. (\%) & & & - \\
\hline Gastric antrum & $125(62.5)$ & - & \\
\hline Cardia & $53(26.5)$ & - & \\
\hline Gastric body & $22(11.0)$ & - & \\
\hline Tumor size $(\mathrm{cm})$, mean $\pm S D$ & $3.2 \pm 1.2$ & - & \\
\hline Pathological grade*, No. (\%) & & & - \\
\hline G1 & $30(15.0)$ & - & \\
\hline G2 & $144(72.0)$ & - & \\
\hline G3 & $26(13.0)$ & - & \\
\hline
\end{tabular}

Table 1 (Continued) 
Table 1 (Continued)

\begin{tabular}{|c|c|c|c|}
\hline Items & Gastric cancer patients $(\mathrm{N}=200)$ & Healthy controls $(\mathrm{N}=200)$ & $P$ value \\
\hline $\mathrm{T} 1$ & $5(2.5)$ & - & \\
\hline $\mathrm{T} 2$ & $17(8.5)$ & - & \\
\hline T3 & $176(88.0)$ & - & \\
\hline N stage, No. (\%) & & & - \\
\hline No & $61(30.5)$ & - & \\
\hline N1 & $48(24.0)$ & - & \\
\hline N2 & $80(40.0)$ & - & \\
\hline I & $22(11.0)$ & - & \\
\hline II & $84(42.0)$ & - & \\
\hline III & $94(47.0)$ & - & \\
\hline Adjuvant chemotherapy, №. (\%) & $133(66.5)$ & - & - \\
\hline Adjuvant radiotherapy, No. (\%) & $26(13.0)$ & - & - \\
\hline
\end{tabular}

*, G1 well differentiation, G2 moderate differentiation, G3 poor differentiation. SD, standard deviation; H. pylori, helicobacter pylori.
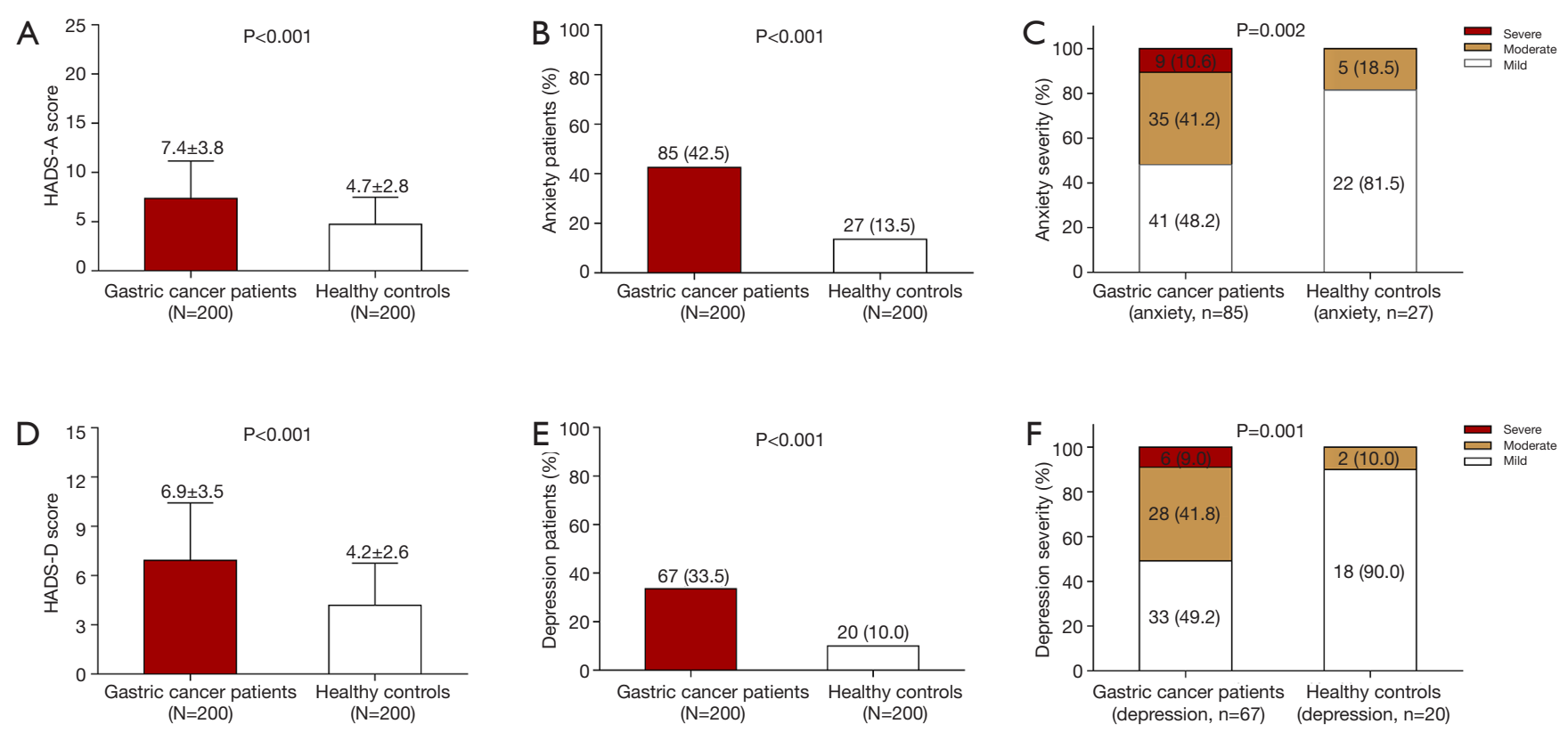

Figure 2 Anxiety and depression in gastric cancer patients and healthy controls. Comparison of HADS-A score (A), anxiety prevalence (B), anxiety severity (C), HADS-D score (D), depression prevalence (E) and depression severity (F) between gastric cancer patients and healthy controls. Comparison was determined by Student's t-test, Chi-square or Wilcoxon rank sum test. $\mathrm{P}<0.05$ was considered significant. HADS-A, Hospital Anxiety and Depression Scale-anxiety; HADS-D, Hospital Anxiety and Depression Scale-depression. 
Table 2 Factors affecting anxiety in gastric cancer patients

\begin{tabular}{|c|c|c|c|c|}
\hline \multirow{2}{*}{ Items } & \multicolumn{4}{|c|}{ Logistic regression model } \\
\hline & $P$ value & OR & Lower & Higher \\
\hline \multicolumn{5}{|l|}{ Univariate logistic regression } \\
\hline Age (>60 years) & 0.883 & 0.960 & 0.548 & 1.682 \\
\hline Smoke & 0.340 & 1.343 & 0.733 & 2.462 \\
\hline Drink & 0.171 & 1.512 & 0.836 & 2.733 \\
\hline Hypertension & 0.029 & 1.972 & 1.072 & 3.628 \\
\hline Hyperlipidemia & 0.205 & 1.508 & 0.799 & 2.848 \\
\hline Married & Reference & - & - & - \\
\hline Single & 0.036 & 2.632 & 1.065 & 6.506 \\
\hline Divorced/widowed & 0.119 & 1.786 & 0.861 & 3.707 \\
\hline Employment status before surgery & 0.871 & 1.049 & 0.587 & 1.875 \\
\hline Higher level of education & 0.785 & 0.952 & 0.670 & 1.353 \\
\hline H. pylori infection (positive) & 0.580 & 0.848 & 0.474 & 1.518 \\
\hline \multicolumn{5}{|l|}{ Tumor location } \\
\hline Gastric antrum & Reference & - & - & - \\
\hline Higher N stage & 0.040 & 1.376 & 1.014 & 1.865 \\
\hline Higher TNM stage & 0.009 & 1.802 & 1.157 & 2.806 \\
\hline \multicolumn{5}{|c|}{ Forward stepwise multivariate logistic regression } \\
\hline Gender (female) & 0.003 & 2.835 & 1.435 & 5.603 \\
\hline Diabetes & $<0.001$ & 6.977 & 2.624 & 18.550 \\
\hline Higher pathological grade & 0.005 & 2.540 & 1.331 & 4.849 \\
\hline Tumor size $(>3 \mathrm{~cm})$ & 0.044 & 1.992 & 1.020 & 3.891 \\
\hline Higher T stage & 0.017 & 3.090 & 1.225 & 7.795 \\
\hline
\end{tabular}

Factors affecting anxiety in gastric cancer patients was analyzed by univariate and forward stepwise multivariate logistic regression model. The explanation of odd ratio, for example, for the factors affecting anxiety in gastric cancer patients, females presented 1.848 times higher risk of anxiety compared with males in gastric cancer patients. OR, odds ratio; Cl, confidence interval; $\mathrm{H}$. pylori, helicobacter pylori. 
Table 3 Factors affecting depression in gastric cancer patients

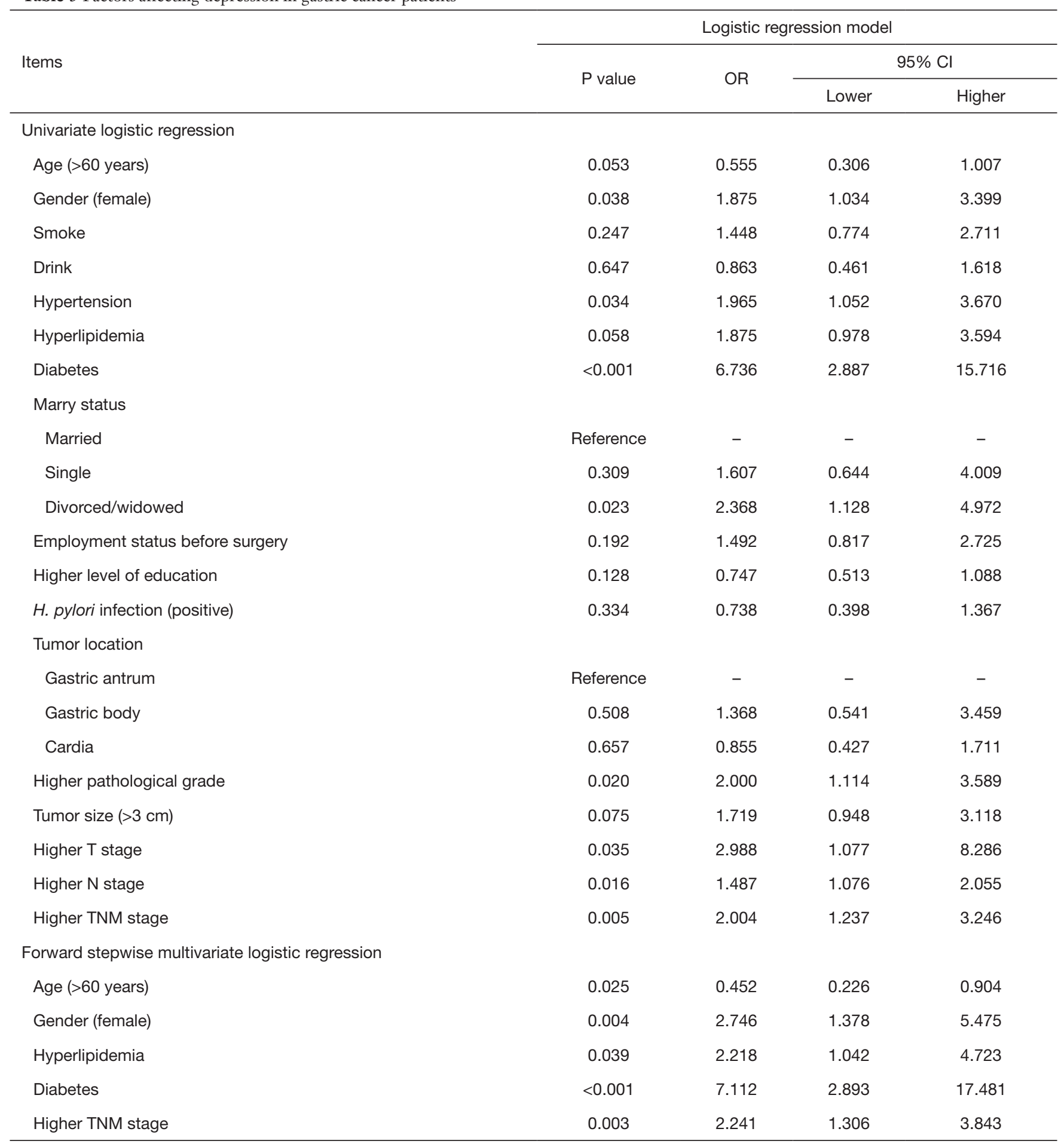

Factors affecting depression in gastric cancer patients was analyzed by univariate and forward stepwise multivariate logistic regression model. OR, odds ratio; $\mathrm{Cl}$, confidence interval; $H$. pylori, helicobacter pylori. 

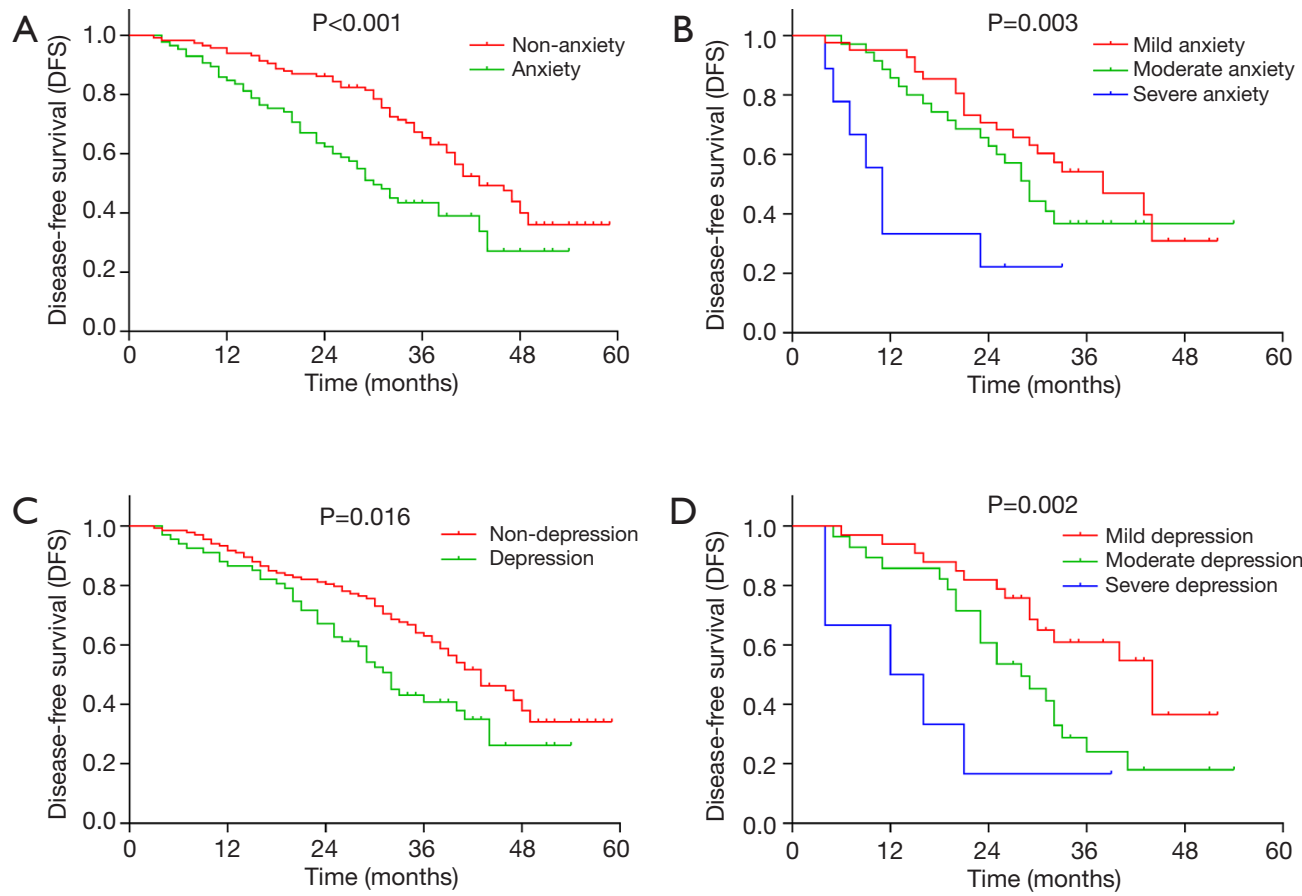

Figure 3 Correlation of DFS with presence and severity of anxiety/depression. (A) Comparison of DFS between gastric cancer patients with non-anxiety and anxiety; (B) comparison of DFS among gastric cancer patients with mild anxiety, moderate anxiety and severe anxiety; (C) comparison of DFS between gastric cancer patients with non-depression and depression. (D) comparison of DFS among gastric cancer patients with mild depression, moderate depression and severe depression. DFS was displayed by Kaplan-Meier curves, and the difference of DFS between/among groups was determined by log-rank test. $\mathrm{P}<0.05$ was considered significant. DFS, disease-free survival.

and then patients with mild anxiety $(\mathrm{P}=0.003)$ (Figure $3 B)$. As for depression, according to the cut-off of 8 assessed by HADS-D, all patients were divided into patients with depression and those without depression (Figure 3C). DFS was reduced in patients with depression compared with patients without depression $(\mathrm{P}=0.016)$. And patients with severe depression had the shortest DFS, followed by patients with moderate depression and then patients with mild depression $(\mathrm{P}=0.002)$ (Figure $3 D)$.

\section{Correlation of anxiety/depression with OS in gastric cancer patients with objective 3}

According to the cut-off of 8 assessed by HADS-A, all patients were divided into patients with anxiety and those without anxiety. OS was decreased in patients with anxiety compared with patients without anxiety $(\mathrm{P}=0.011)$ (Figure $4 A$ ). Meanwhile, patients with severe anxiety had the shortest OS, followed by patients with moderate anxiety and then patients with mild anxiety $(\mathrm{P}<0.001)$ (Figure $4 B)$. Regarding depression, according to the cut-off of 8 assessed by HADS-D, all patients were divided into patients with depression and those without depression. OS was decreased in patients with depression compared with patients without depression $(\mathrm{P}=0.022)$ (Figure $4 C)$, and patients with severe depression had the shortest OS, followed by patients with moderate depression and then patients with mild depression $(\mathrm{P}<0.001)$ (Figure 4D).

\section{Correlation of adjuvant chemotherapy/radiotherapy with common complications}

In gastric cancer patients, there was no association between adjuvant chemotherapy with hypertension $(\mathrm{P}=0.404)$, hyperlipidemia $(\mathrm{P}=0.589)$, or diabetes $(\mathrm{P}=0.279)$ (Table S1). In addition, there was also no association between adjuvant radiotherapy with hypertension $(\mathrm{P}=0.671)$, hyperlipidemia $(\mathrm{P}=0.399)$, or diabetes $(\mathrm{P}=0.986)$. 

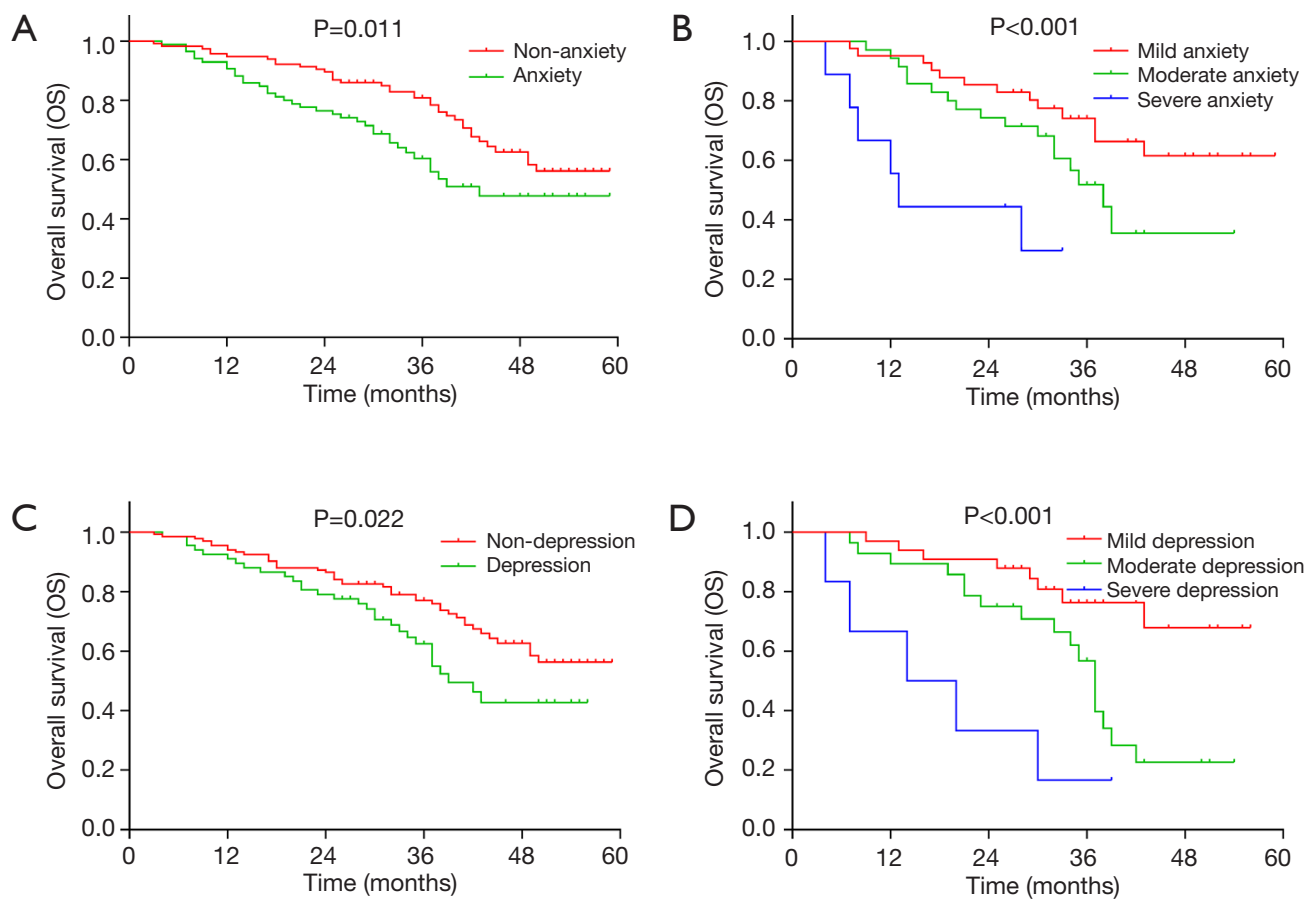

Figure 4 Correlation of OS with presence and severity of anxiety/depression. (A) Comparison of OS between gastric cancer patients with non-anxiety and anxiety; (B) comparison of OS among gastric cancer patients with mild anxiety, moderate anxiety and severe anxiety; (C) comparison of OS between gastric cancer patients with non-depression and depression; (D) comparison of OS among gastric cancer patients with mild depression, moderate depression and severe depression. OS was displayed by Kaplan-Meier curves, and the difference of OS between/among groups was determined by log-rank test. $\mathrm{P}<0.05$ was considered significant. OS, overall survival.

\section{Discussion}

The present study aimed to explore the prevalence, risk factors and prognostic role of anxiety and depression in surgical gastric cancer patients, and observed that: (I) prevalence and severity of anxiety as well as depression were greatly elevated in gastric cancer patients compared with healthy controls. (II) Gender (female), diabetes, higher pathological grade, larger tumor size, higher $\mathrm{T}$ stage were independent predictive factors for higher risk of anxiety, and age ( $>60$ years), gender (female), hyperlipidemia, diabetes and higher TNM stage were independent predictive factors for higher risk of depression in gastric cancer patients. (III) Anxiety/depression and the severity of anxiety/depression were negatively associated with DFS and OS in gastric cancer patients.

Emerging evidence reveals the high prevalence of anxiety and depression in patients with various types of cancers after diagnosis, which are of considerable importance, have adverse influence on the quality of lives and result in a long-term burden on patients as well as their families
(12-15). One study with a large, representative sample of cancer patients after diagnosis indicates that $19.0 \%$ and $12.9 \%$ of cancer patients showed clinical levels of anxiety and depression respectively (12). Another study shows that overall anxiety and depression symptoms is observed in $18.3 \%$ and $24.0 \%$ of cancer patients, and among all the cancer patients, patients with gastric cancer have higher rate of mixed anxiety/depression symptoms (13). In addition, one study recruiting 452 patients with the most common cancers (including gastric cancer) indicates that $39.2 \%$ cancer patients have slight levels of anxiety/depression (16). However, majority of studies explore the prevalence of anxiety and depression in gastric cancer patients who have been received treatment of chemotherapy or surgery, and the prevalence of preoperative anxiety and depression in gastric cancer patients is limited explored. Therefore, the present study was conducted, which revealed that the prevalence of anxiety and depression were $42.5 \%$ and $33.5 \%$ in surgical gastric cancer patients before surgery, and these gastric cancer patients presented increased prevalence and 
increased severity of anxiety and depression compared with healthy controls. The possible reasons might include that: (I) the diagnosis and apprehension about surgery might contribute to psychological burden, which might cause the high prevalence and advanced severity of anxiety/depression in preoperative gastric cancer patients. (II) Furthermore, the adverse effect of gastric cancer, including the following loss of gastric function, financial stress from resection surgery, and the fear of cancer reoccurrence might increase the risk and severity of anxiety/depression in preoperative gastric cancer patients.

Several studies further investigate the risk factors for anxiety and depression among patients with gastric cancer $(9,15,17)$. One study in Korea investigates the risk factor affecting psychiatric comorbidity including anxiety and depression in patients with newly diagnosed cancers, indicates that older age and gender (female) are observed to be risk factors for psychiatric comorbidity (15). Another population-based study in Taiwan discloses that female gender and hypertension are the independent predictive variables for developing subsequent depressive disorder in gastric cancer patients (18). In addition, distressed personality, which is defined as the combination of negative affectivity and social inhibition, is associated with increased levels of anxiety and depression (19). These previous studies exhibit factors affecting psychiatric disorder in gastric cancer patients after diagnosis, while, to be specific, for pre-operative gastric cancer patients, the factors affecting the risk of anxiety/depression might be different. Thus, the influence of socio-demographic characteristics, complications, tumor features on anxiety/depression was assessed in the present study, which exhibited that gender (female), diabetes, higher pathological grade, larger tumor size, higher $\mathrm{T}$ stage were independent predictive factors for increased risk of anxiety, and age ( $>60$ years), gender (female), hyperlipidemia, diabetes and higher TNM stage were independent predictive factors for increased risk of depression in gastric cancer patients. The possible reasons might include that: (I) gastric cancer patients with advanced tumor features, including higher pathological grade, larger tumor size, higher T stage and higher TNM stage, which might endure stronger fear to disease and treatment, which contributed to higher risk of anxiety/depression. (II) Female cancer patients were reported to have lower levels of emotional support compared with male cancer patients and less emotional support was correlated with higher risk of anxiety/depression (20). Thus, female gastric cancer patients were more vulnerable to anxiety/depression compared with males. (III) Additionally, older patients had worse ability of physical recovery and adaptation compared with younger patients, which might lead to highly prevalent depression. (IV) Furthermore, patients with complications, such as diabetes, hyperlipidemia, might have increased healthrelated burden, contributing to the higher risk of anxiety/ depression.

Existing evidences illustrate that psychological distress has an unfavorable prognostic impact on cancer patients $(9,13,19,21,22)$. For example, one Korean study indicates that gastric cancer patients with psychological distress have worse DFS and OS compared with those without psychological distress (9). However, the research investigating the effect of anxiety/depression on prognosis for surgical gastric cancer patients is rare in China. In the present study, the prevalence and severity of anxiety/ depression were negatively associated with DFS and OS in gastric cancer patients. The possible explanation might include that: (I) the compliance to adjuvant chemotherapy might be worse in patients with anxiety/depression, which was responsible for the poor survival in patients with anxiety/depression. (II) Patients with anxiety/depression might have problem of insomnia, pain, reflux, dyspnea, etc., contributing to undesirable recovery from cancer and further poor prognosis in surgical gastric cancer patients. (III) According to the previous finding, advanced tumor features (including higher pathological grade, higher TNM stage) were associated with higher risk of anxiety/ depression, thus gastric cancer patients with anxiety/ depression might have worse prognosis via interaction with these tumor features. These findings in the present study implied the necessity of screening for anxiety/depression, a psychotherapeutic approach, and an adapted psychologicalcentered care program which included rehabilitation of emotional disorders, education of gastric cancer, surveillance of tumor reoccurrence and metastasizes, etc., which could relieve the anxiety and depression, and further improve the long-term clinical outcomes in surgical gastric cancer patients.

There were several limitations in the present study. Firstly, the rating scales (HADS score) rather than clinical diagnostic interviews was used, to identify anxiety and depression, therefore some real conditions of the gastric cancer patients following the cancer diagnosis might not be reflect to some extent, which needed to be exploration by further studies. Secondly, postoperative treatments for gastric cancer patients in follow-up period were different, which might lead to variation in survival. Thirdly, anxiety 
and depression status were assessed by single scale (HADS), and more anxiety and depression evaluation scales were needed to improve the validation. Fourthly, since the patients enrolled did not receive the neo-adjuvant treatments, therefore the association of neo-adjuvant treatment with anxiety/depression needed further study for validation.

In conclusion, anxiety/depression are common and severe, which predict unfavorable prognosis in surgical gastric cancer patients, meanwhile gender (female), advanced tumor features, diabetes and hyperlipidemia are independent predictive factors for higher risk of anxiety and depression. Therefore, the frequent screening of these risk factors is essential to prevent anxiety/depression after surgery, which would further improve prognosis and psychological well-being in surgical gastric patients.

\section{Acknowledgments}

I am extremely grateful for my colleagues for their support in assessment of anxiety and depression and collection of follow-up data during the whole study.

Funding: None.

\section{Footnote}

Conflicts of Interest: The author has completed the ICMJE uniform disclosure form (available at http://dx.doi. org/10.21037/tcr.2020.01.11). The author has no conflicts of interest to declare.

Ethical Statement: The author is accountable for all aspects of the work in ensuring that questions related to the accuracy or integrity of any part of the work are appropriately investigated and resolved. This study was approved by the Ethics Committee of the 2nd Affiliated Hospital of Harbin Medical University (No. Ky-2014-211) and conducted in accord with the Declaration of Helsinki (as revised in 2013). All participants signed the informed consents before they participated in the study.

Open Access Statement: This is an Open Access article distributed in accordance with the Creative Commons Attribution-NonCommercial-NoDerivs 4.0 International License (CC BY-NC-ND 4.0), which permits the noncommercial replication and distribution of the article with the strict proviso that no changes or edits are made and the original work is properly cited (including links to both the formal publication through the relevant DOI and the license). See: https://creativecommons.org/licenses/by-nc-nd/4.0/.

\section{References}

1. Bray F, Ferlay J, Soerjomataram I, et al. Global cancer statistics 2018: GLOBOCAN estimates of incidence and mortality worldwide for 36 cancers in 185 countries. CA Cancer J Clin 2018;68:394-424.

2. Thrift AP, El-Serag HB. Burden of Gastric Cancer. Clin Gastroenterol Hepatol 2020;18:534-42.

3. Song $Z, W u$ Y, Yang J, et al. Progress in the treatment of advanced gastric cancer. Tumour Biol 2017;39:1010428317714626.

4. Li T, Xiang PLB. Therapeutic effects of endoscopic mucosal resection on the recovery and prognosis of early gastric cancer. J BUON 2019;24:1087-91.

5. Groene P, Eisenlohr J, Zeuzem C, et al. Postoperative nausea and vomiting in bariatric surgery in comparison to non-bariatric gastric surgery. Wideochir Inne Tech Maloinwazyjne 2019;14:90-5.

6. Pedziwiatr M, Mavrikis J, Witowski J, et al. Current status of enhanced recovery after surgery (ERAS) protocol in gastrointestinal surgery. Med Oncol 2018;35:95.

7. Zhang P, Lan TH, Zhou YM, et al. Risk factor analysis of perioperative complications in patients with radical gastrectomy for gastric cancer. Zhonghua Wei Chang Wai Ke Za Zhi 2019;22:736-41.

8. Matsushita T, Matsushima E, Maruyama M. Anxiety and depression of patients with digestive cancer. Psychiatry Clin Neurosci 2005;59:576-83.

9. Kim GM, Kim SJ, Song SK, et al. Prevalence and prognostic implications of psychological distress in patients with gastric cancer. BMC Cancer 2017;17:283.

10. Gorrepati VS, Yadav S, Stuart A, et al. Anxiety, depression, and inflammation after restorative proctocolectomy. Int J Colorectal Dis 2018;33:1601-6.

11. Zigmond AS, Snaith RP. The hospital anxiety and depression scale. Acta Psychiatr Scand 1983;67:361-70.

12. Linden W, Vodermaier A, Mackenzie R, et al. Anxiety and depression after cancer diagnosis: prevalence rates by cancer type, gender, and age. J Affect Disord 2012;141:343-51.

13. Brintzenhofe-Szoc KM, Levin TT, Li Y, et al. Mixed anxiety/depression symptoms in a large cancer cohort: prevalence by cancer type. Psychosomatics 2009;50:383-91.

14. Xu L, Pan Q, Lin R. Prevalence rate and influencing 
factors of preoperative anxiety and depression in gastric cancer patients in China: Preliminary study. J Int Med Res 2016;44:377-88.

15. Lee BO, Choi WJ, Sung NY, et al. Incidence and risk factors for psychiatric comorbidity among people newly diagnosed with cancer based on Korean national registry data. Psychooncology 2015;24:1808-14.

16. Su M, Hua X, Wang J, et al. Health-related quality of life among cancer survivors in rural China. Qual Life Res 2019;28:695-702.

17. Han KH, Hwang IC, Kim S, et al. Factors associated with depression in disease-free stomach cancer survivors. J Pain Symptom Manage 2013;46:511-22.

18. Hu LY, Liu CJ, Yeh CM, et al. Depressive disorders among patients with gastric cancer in Taiwan: a nationwide population-based study. BMC Psychiatry 2018;18:272.

19. Zhang JK, Fang LL, Zhang DW, et al. Type D Personality in Gastric Cancer Survivors: Association With Poor Quality of Life, Overall Survival, and Mental Health. J Pain Symptom Manage 2016;52:81-91.

20. Ghazavi-Khorasgani Z, Ashrafi-Rizi H, Mokarian F, et al. Health information seeking behavior of female breast cancer patients. J Educ Health Promot 2018;7:138.

21. Di Giacomo D, Ranieri J, Guerra F, et al. Survivorship in young women after early breast cancer: a cross-sectional study of emotional traits along 3-years perspective. Riv Psichiatr 2019;54:160-7.

22. Yu H, Wang Y, Ge X, et al. Depression and survival in Chinese patients with gastric cancer: a prospective study. Asian Pac J Cancer Prev 2012;13:391-4.
Cite this article as: Han L. Prevalence, risk factors and prognostic role of anxiety and depression in surgical gastric cancer patients. Transl Cancer Res 2020;9(3):1371-1383. doi: 10.21037/tcr.2020.01.11 
Supplementary

Table S1 Correlations of adjuvant treatments with common complications

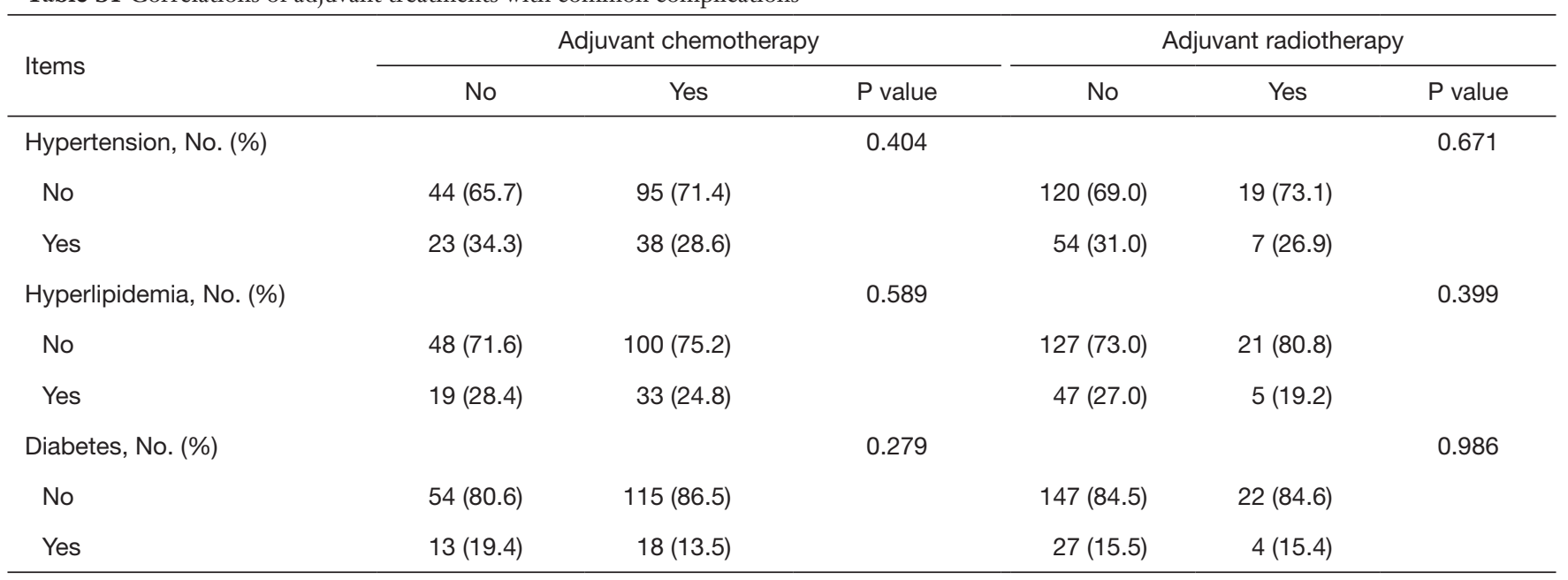

Comparison was determined by Chi-square test. 